Revista Destaques Acadêmicos, Lajeado, v. 12, n. 3, 2020. ISSN 2176-3070

DOI: http://dx.doi.org/10.22410/issn.2176-3070.v12i3a2020.2594

http://www.univates.br/revistas

\title{
COMPARAÇÃO ENTRE AVALIAÇÕES FUNCIONAIS DE MEMBROS INFERIORES PRÉ E PÓS-PERÍODO DE PREPARAÇÃO EM ATLETAS DE VOLEIBOL FEMININO DE BASE
}

\author{
Bruna Schlabitz Battisti ${ }^{1}$, Rodrigo Lara Rother ${ }^{2}$
}

\begin{abstract}
Resumo: O voleibol de rendimento evoluiu em diversos aspectos e, um deles, é a exigência física, que chegou a um patamar que não envolve somente o desejo de jogar bem, mas sim, a soma de diversos requisitos associados às técnicas de treinamento. Em uma partida de voleibol acontecem de 250 a 300 atos motores, representados nos saltos, corridas e quedas. Esta gama variada de exigências, associada à desequilíbrios físicos e/ou posturais, pode levar o atleta a desenvolver algum tipo de lesão. O objetivo do presente estudo é comparar as avaliações funcionais realizadas no pré e pós período de preparação em uma equipe de voleibol de base feminina. A metodologia empregada caracteriza-se como quantitativa, descritiva e transversal, utilizando informações do banco de dados de uma equipe de voleibol feminino, que aplicou o Y Balance Test (YBT) em 12 atletas da categoria infanto juvenil. Os resultados foram tabulados em uma planilha Excel, na qual foi calculado o score composto, descritos por meio de média e desvio padrão, e comparados a partir do Teste T de Student pareado. A equipe obteve como média no período pré preparação o score composto para MID 96,37 (dp 5,26) e para MIE 97,62 (dp 6,25). Dentre as atletas avaliadas, apenas uma $(8,33 \%)$ apresentou risco de lesão nas duas avaliações realizadas (pré-temporada e pós-preparação), 3,5 vezes maior que suas colegas de equipe, com valores de 87,81 para MID e 85,10 para MIE. Conclui-se que o risco de lesões da equipe avaliada é baixo e que as avaliações funcionais de pré e pós-preparação são fundamentais no auxílio à prevenção de lesões de membros inferiores no voleibol.
\end{abstract}

Palavras-chave: Treinamento Esportivo. Avaliação Funcional. Voleibol. Lesões.

1 Graduada do Curso de Educação Física na Universidade do Vale do Taquari UNIVATES Lajeado/RS. bruna.s.battisti@gmail.com

2 Doutor em Ambiente e Desenvolvimento, docente do curso de Educação Física da Universidade do Vale do Taquari UNIVATES - Lajeado/RS. rodrigorother@univates.br 


\section{INTRODUÇÃO}

O voleibol evoluiu em diversos aspectos desde sua criação. As regras vêm sendo modificadas para que a modalidade se torne cada vez mais atrativa e dinâmica. As exigências técnicas e táticas beiram a perfeição na realização das ações e, fisicamente, nunca se viu antes na história atletas tão altos e com tanta capacidade motora (ANTÔNIO; SANTOS, 2012).

O aspecto físico talvez seja o que mais chama a atenção nesta evolução. Com destaque para as capacidades de força e potência de membros inferiores, já que é imposta a necessidade da realização de inúmeros deslocamentos curtos, velozes e uma grande quantidade de saltos realizados pelos atletas durante os treinamentos e competições (LOMBARDI et al., 2011). Corroboram com essa percepção Franca, Fernandes e Cortez (2004), quando afirmam que o alto nível de desempenho gera sobrecargas corporais intensas com aplicação de força muscular, grandes amplitudes articulares e transferências de peso quase superiores à capacidade humana.

Diante disso, a possibilidade de ocorrência de lesões tem gerado muita preocupação por parte dos profissionais que trabalham com o voleibol e nos próprios atletas, que percebem suas carreiras em risco. Segundo Hootman et al. (2007), 50\% das lesões do voleibol ocorrem em membros inferiores, sendo que $30 \%$ do total se apresentam como entorses e lesões ligamentares de joelho e tornozelo. Jadhav et al. (2010) identificaram que uma das principais lesões causadas pelo voleibol é na articulação do tornozelo, representando $23 \%$ das ocorrências. Dizem ainda os autores citados acima, que esta articulação sofre altas pressões quando ocorrem saltos e aterrissagens como os de defesa, armação de jogadas e os movimentos de ataque. Seguindo com o autor Canavan (2001), as lesões no tornozelo representam $25 \%$ das contusões no voleibol. Ainda, Pacheco et al. (2000) e Farina (2008) ressaltam que as entorses por inversão do tornozelo são as lesões mais comuns do esporte, também sendo responsável por aproximadamente $25 \%$ das lesões esportivas e, inclusive, resulta em um grau de instabilidade funcional devido a redução das forças dos músculos eversores da articulação e da sensibilidade proprioceptivas.

Para evitar a ocorrência de lesões, profissionais integrantes das comissões técnicas atuam na aplicação de testes e avaliações de parâmetros que possam ser indicadores de possíveis lesões futuras. Tubino e Moreira (2003) apresentam as avaliações funcionais como importantes preditores de lesão. Estas avaliações consistem em analisar os padrões fundamentais de movimento e identificar alterações na mobilidade, estabilidade e assimetrias. A correção destes padrões, uma vez conseguida, permite maior eficiência de movimento para o atleta, influenciando em uma melhora no desempenho e diminuição do potencial de lesões (SILVA; ALMEIDA; REGIS, 2017).

Gomes (2009) afirma que o momento mais indicado para que estas avaliações ocorram é antes ou logo no início da temporada, onde os atletas estão 
retornando aos treinamentos e podem ser rastreados possíveis fatores de risco de lesões e combatidos com treinamento preventivo. Para Matveiev (1991), um dos precursores da periodização no esporte, a prescrição do treinamento deve ser organizada visando uma sequência lógica, que harmonize os conteúdos físicos com os técnicos, táticos e psicológicos. O autor salienta que, no modelo de Periodização Clássica, há um período chamado de Período de Preparação, que caracteriza-se por utilizar maior volume e menor intensidade nos treinamentos, participação em poucas ou nenhuma competição, estímulos às capacidades físicas básicas e a implementação de estratégias visando a prevenção de lesões. Maffey e Emery (2006) comentam que as ações indicadas por Matveiev para serem realizadas neste período inicial da temporada são comunmente aplicadas no esporte de alto rendimento.

Cotta et al. (2008) citam a eficiência, o baixo custo e a facilidade na prática da aplicação dos testes como fundamentais para sua realização no esporte. Desta forma, o Y Balance Test (YBT) apresenta-se como uma opção coerente às necessidades citadas por estes autores.

O YBT tem por característica identificar os desequilíbrios funcionais dos membros inferiores e exige controle postural e proprioceptivo, força muscular e amplitude de movimento da articulação. Além disso, é uma ferramenta de triagem funcional desenvolvida para avaliar a estabilidade dinâmica dos membros inferiores, monitorar o progresso da reabilitação, avaliar os desequilíbrios funcionais após a lesão e identificar atletas com alto risco de lesão, e, igualmente, caracteriza-se como opção para avaliar o equilíbrio postural, identificar deficiências do equilíbrio dinâmico, avaliar efeitos dos treinamentos em atletas saudáveis, e adequado para ser aplicado em pessoas fisicamente ativas, oferecendo medidas confiáveis (HERTEL et al., 2006).

Dallinga, Benjaminse, Lemmink (2012) realizaram uma revisão sistemática com intuito de encontrar ferramentas que poderiam prever o risco de lesões em membros inferiores nos esportes de equipe. Esses autores constaram que o teste funcional do YBT apresenta uma sensibilidade apurada para discriminar jovens do sexo feminino a desenvolverem futuras lesões. Outros estudos mostraram ainda que este teste apresenta boa sensibilidade para indicar déficits posturais de acordo com os autores Hertel et al. (2006) e Mancini, Horak (2010).

Considerando todos estes aspectos, o objetivo do presente estudo é comparar os desequilíbrios funcionais de membros inferiores nas avaliações pré-temporada e pós-período de preparação em uma equipe de voleibol de base feminina.

\section{PROCEDIMENTOS METODOLÓGICOS}

O estudo caracteriza-se como quantitativo, descritivo e comparativo. A partir desta abordagem, foram coletadas informações no banco de dados de 
uma equipe de voleibol feminino de base do Rio Grande do Sul, composta por 12 atletas, com idade média de 16,5 anos. Essa equipe participa de competições internacionais e tem revelado em sua história inúmeras atletas que hoje disputam a Superliga nacional e atuam fora do país (Estados Unidos e Europa).

Os treinamentos desta equipe são periodizados segundo o modelo de Periodização Clássico, proposto por Matveiev (1991), realizando um Período de Preparação no início da temporada, caracterizado por treinamentos de maior volume em relação a intensidade. A equipe treina de três a cinco vezes na semana, com cargas onduladas. A média semanal de horas de treinamento são de 12, distribuídas em treinamento técnico tático (nove horas) e físico (três horas). O treinamento físico ocorre na academia (musculação para treino de força e potência) e na quadra (exercícios funcionais de propriocepção, equilíbrio, flexibilidade, resistência muscular localizada e agilidade), sendo esse segundo local o mesmo do treinamento técnico/tático.

O Período de Preparação ocorreu do dia três de março a 11 de maio de 2018, sendo estes dias marcados pela realização de avaliações funcionais. Estas avaliações foram denominadas como "Pré-preparação" e "Pós-preparação".

Dentre os testes realizados pela equipe e disponíveis no banco de dados, foi escolhido o YBT, pois representava a avaliação mais condizente com a predição de lesões de membros inferiores. $\mathrm{O}$ qual apresenta a capacidade de quantificar o desempenho do atleta de acordo com a distância durante os alcances realizados na direção Anterior (A), Póstero Lateral (PL) e Póstero Medial (PM), enquanto realizam equilíbrio uni podal. Essas direções condizem com os planos de movimento (frontal, transverso e sagital) e, portanto, apresenta formato da letra Y. De acordo com a descrição contida no banco de dados, o procedimento de avaliação do YBT foi composto por três fitas métricas coladas no chão com fita adesiva e separadas por um goniômetro em um ângulo de 45 graus. Com as atletas descalças e em pé no centro do Y em apoio uni podal, com as mãos na cintura e com o membro inferior que não estava apoiado no solo, tocava sutilmente a distância máxima alcançada com a ponta do pé sobre a fita métrica, sendo que o membro a ser avaliado era o qual mantinha-se fixo no solo, e não o membro que movia-se nas três direções do teste. As medidas foram realizadas na direção anterior com o membro inferior direito e esquerdo, seguindo pelas outras direções: póstero lateral e póstero medial, sendo realizadas três medidas para cada membro em cada direção. A avaliada retornava então para a posição inicial, enquanto mantinha o equilíbrio. As instruções, tanto verbais, como visuais, de como funcionaria o teste, foram dadas a todos os sujeitos antes da avaliação. Além disso, cada atleta realizou três experimentações da prática em cada uma das direções, para que fossem familiarizadas com o teste.

O YBT era interrompido nos casos em que o pé de apoio fosse movido e a mão fosse retirada da cintura, sendo novamente posicionado o sujeito, e repetia-se o teste, além de quando a comissão técnica percebia que o pé que 
deveria alcançar a maior distância apoiasse de forma não sutil à fita e/ou quando a atleta se desequilibrasse em qualquer ponto durante a execução.

A máxima das três distâncias alcançadas por cada membro inferior e em cada uma das três direções foi anotada para posteriormente calcular o Score Composto, um valor expressado em percentual que considera todas as direções do movimento usadas no YBT. Desta forma, realizou-se uma análise de todas as distâncias em centímetros alcançadas pelas atletas, validadas pela comissão técnica e, em seguida, registradas em uma planilha. Além disso, foi realizada a medição do comprimento dos membros inferiores, onde cada atleta era avaliada individualmente. Estas foram instruídas a permanecer deitadas em uma superfície plana e estável (maca) para que se concretizasse a coleta. A medida foi realizada pela distância entre a espinha ilíaca ântero superior e o maléolo medial, e, após, eram anotadas as distâncias em centímetros na ficha correspondente à avaliada. O Score Composto final é baseado na divisão entre a soma da distância máxima de alcance nas direções anterior, póstero medial e póstero lateral, por três vezes o comprimento do membro inferior da atleta e, por fim, multiplicado por cem: [(Máx. Anterior + Máx. Póstero Medial + Máx Póstero Lateral) / (Comp. Perna x 3)] x100 (FILIPA et al., 2010).

Os resultados do YBT foram tabulados em planilha Excel e descritos por meio de média e desvio padrão. A comparação entre as médias foi realizada por meio de Teste T de Student pareado, adotando um nível de significância de $\mathrm{p}<0,05$.

Esta pesquisa foi aprovada pelo Comitê de Ética em Pesquisa (COEP) da Universidade do Vale do Taquari - UNIVATES, sob parecer número 2.781.529.

\section{APRESENTAÇÃO DOS RESULTADOS}

Os dados coletados com a utilização do YBT na avaliação pré e póspreparação, bem como o cálculo do Score Composto e as diferenças existentes entre os valores do YBT encontrados nos MID e MIE, podem ser observados na Tabela 1. 
Tabela 1 - Médias dos resultados do YBT, Score Composto e Diferenças entre MMII direito e esquerdo nas avaliações Pré e Pós-período de preparação, em equipe feminina de voleibol de base.

\begin{tabular}{|c|c|c|c|c|c|c|c|c|c|c|c|}
\hline \multirow{2}{*}{ PERÍODO } & \multicolumn{6}{|c|}{ Y BALANCE TEST PROPRIOCEPÇÃO (cm) } & \multirow{2}{*}{$\begin{array}{c}\text { Score } \\
\text { Comp. } \\
\text { D (\%) }\end{array}$} & \multirow{2}{*}{$\begin{array}{c}\text { Score } \\
\text { Comp. } \\
\text { E } \\
(\%)\end{array}$} & \multirow{2}{*}{$\begin{array}{l}\text { AN } \\
\text { D-E } \\
(\mathrm{cm})\end{array}$} & \multirow{2}{*}{$\begin{array}{c}\text { Diferenças } \\
\text { PM } \\
\text { D-E } \\
(\mathrm{cm})\end{array}$} & \multirow{2}{*}{$\begin{array}{l}\text { PL } \\
\text { D-E } \\
(\mathrm{cm})\end{array}$} \\
\hline & D-AN & D-PM & D-PL & E-AN & E-PM & E-PL & & & & & \\
\hline $\begin{array}{l}\text { Pré Prep. } \\
\text { (dp) }\end{array}$ & $\begin{array}{l}84,67 \\
(5,79)\end{array}$ & $\begin{array}{c}89,50 \\
(5,4)\end{array}$ & $\begin{array}{l}88,08 \\
(7,56)\end{array}$ & $\begin{array}{l}84,25 \\
(5,36)\end{array}$ & $\begin{array}{l}90,00 \\
(7,52)\end{array}$ & $\begin{array}{l}91,58 \\
(6,53)\end{array}$ & $\begin{array}{l}96,37 \\
(5,26)\end{array}$ & $\begin{array}{l}97,62 \\
(6,25)\end{array}$ & $\begin{array}{c}0,42 \\
(3,15)\end{array}$ & $\begin{array}{c}0,5 \\
(3,94)\end{array}$ & $\begin{array}{c}3,5 \\
(5,47)\end{array}$ \\
\hline $\begin{array}{l}\text { Pós Prep. } \\
\text { (dp) }\end{array}$ & $\begin{array}{l}85,17 \\
(4,57)\end{array}$ & $\begin{array}{l}87,25 \\
(5,67)\end{array}$ & $\begin{array}{l}92,17 \\
(5,84)\end{array}$ & $\begin{array}{l}84,83 \\
(4,13)\end{array}$ & $\begin{array}{l}88,00 \\
(6,51)\end{array}$ & $\begin{array}{l}94,00 \\
(5,58)\end{array}$ & $\begin{array}{l}97,33 \\
(5,77)\end{array}$ & $\begin{array}{l}97,88 \\
(6,38)\end{array}$ & $\begin{array}{c}0,33 \\
(2,81)\end{array}$ & $\begin{array}{c}0,75 \\
(4,41)\end{array}$ & $\begin{array}{r}1,83 \\
(3,61)\end{array}$ \\
\hline Teste T (p) & 0,33 & 0,12 & 0,04 & 0,26 & 0,12 & 0,09 & 0,27 & 0,17 & 0,44 & 0,15 & 0,11 \\
\hline
\end{tabular}

$\mathrm{D}-\mathrm{AN}=$ Anterior com membro direito; $\mathrm{D}-\mathrm{PL}=$ Póstero lateral com membro direito; $\mathrm{D}-\mathrm{PM}=$ Póstero medial com membro direito; E-AN= Anterior com membro esquerdo; E-PM= Póstero medial com membro esquerdo. E-PL= Póstero lateral com membro esquerdo.

Fonte: Os autores (2018).

Para classificação de risco de lesões, foram seguidas as recomendações dos autores Butler et al. (2013), Smith, Chimera e Warren (2015) e Gonell, Romero e Soler (2015), Gribble, Hertel e Plisky (2012), os quais citam em seus estudos que atletas que apresentam Scores Compostos menores que 89,6\% tem risco de lesão aumentado em 3,5 vezes. Os mesmos autores alertam que, atletas que apresentam diferença entre valores de MID e MIE maiores que $4 \mathrm{~cm}$ ou $6 \%$, tem chances triplicadas de ocorrência de lesões.

Os valores aqui obtidos para o YBT foram utilizados para cálculo do Score Composto. Para esta variável, a avaliação pré-preparação apresentou como média de membro inferior direito (MID) valor de $96,37 \%$ (dp 5,26) e para membro inferior esquerdo (MIE) $97,62 \%$ (dp 6,25). Dentre as atletas avaliadas, apenas uma apresentou os valores mais baixos que o recomendado pela literatura para um valor seguro, tanto para MID (87,81\%) quanto para MIE $(85,10 \%)$. Além disso, apenas uma atleta apresentou diferença classificada como de risco (6,05\%) quando comparados Score Composto de MID e MIE. Já quando comparados os valores de YBT para as três avaliações de MID e MIE, sete atletas apresentaram diferenças maiores que $4 \mathrm{~cm}$ em pelo menos uma das três direções avaliadas no teste.

A avaliação pós-preparação apresentou como média do Score Composto de MID valor de $97,33 \%$ (dp 5,77) e para MIE 97,88\% (dp 6,38). Dentre as atletas avaliadas, uma única atleta (a mesma da avaliação anterior) apresentou o valor mais baixo que o recomendado pela literatura, mas desta vez somente para MIE $(85,10 \%)$. Na avaliação deste período quatro atletas apresentaram diferença classificada como de risco quando comparados Score Composto de MID e MIE $(9,17 \% ; 7,69 \% ; 7,15 \% ; 6,23 \%)$. Já quando comparados os valores de YBT para as 
três avaliações de MID e MIE, cinco atletas apresentaram diferenças maiores que $4 \mathrm{~cm}$ em pelo menos uma das três direções avaliadas no teste.

Comparando todas variáveis avaliadas entre os períodos de pré e póspreparação, a análise estatística encontrou diferença significativa somente para D-PL $(\mathrm{p}=0,04)$ no sentido de melhora no alcance do YBT a favor da póspreparação. Também vale ressaltar que diminuíram de sete (pré) para cinco (pós) as atletas que apresentaram diferenças maiores que $4 \mathrm{~cm}$ entre MID e MIE no YBT e a atleta que havia valores baixos em ambos MI, melhorou em pelo menos um deles.

\section{DISCUSSÃO}

Partindo dos estudos de Marwan et al. (2012) se percebe o voleibol como a modalidade em que mais ocorrem lesões de membros inferiores $(73,1 \%)$ e articulares $(43,6 \%)$ quando comparado aos outros esportes com bola. Ainda assim, na equipe de voleibol investigada, somente uma atleta apresentou situação de risco, para MID e MIE na avaliação pré-preparação e MIE na póspreparação. Quanto às diferenças MID e MIE nas avaliações do YBT, na prépreparação sete atletas apresentaram valores de riscos acima dos apresentados pela literatura e na avaliação pós-preparação cinco atletas apresentaram esse risco.

Estudos de Plisky et al. (2006) encontraram valores de desempenho de 235 atletas universitários de basquete do sexo masculino e feminino durante a temporada de 2004 e 2005. Atletas do sexo masculino que tiveram assimetria maior que $4 \mathrm{~cm}$ na direção anterior tiveram 2,5 vezes mais chances de se lesionarem durante a temporada. Já as atletas do sexo feminino em que o score composto foi menor que $94 \%$ tiveram 6,5 vezes mais risco. Ainda os autores Gribble, Hertel e Plisky (2012) complementam que até $6 \%$ de diferença entre os Scores Compostos de MI ou diferença de $4 \mathrm{~cm}$ entre os mesmos são consideradas dentro dos padrões, ou seja, acima destes valores há probabilidade de ocorrerem lesões.

Os autores Correa e Linden Junior (2016) avaliaram, em 20 atletas amadoras de voleibol, o controle postural dinâmico antes e após treinamento, onde os resultados não apresentaram variações significativas no controle postural dinâmico antes e após o treino, tendo assim semelhança entre os valores e comparações apresentados neste estudo com os salientados pela literatura.

Hale, Hertel e Olmsted-kramer (2007) descreveram a utilização de um teste (SEBT) que caracteriza-se semelhante ao YBT. Estes autores estudaram o pré e pós teste em indivíduos submetidos a um período de quatro semanas de tratamento, devido a instabilidade crônica de tornozelo. Na avaliação dos valores médios, observou-se diferença significativa no Score Composto ao comparar o lado lesionado $(70,54 \pm 7,70 \mathrm{~cm})$ com o lado sadio $(67,45 \pm 7,69 \mathrm{~cm}$, 
$\mathrm{p}=0,0003)$. Fato não observado para o grupo controle ao comparar o membro inferior direito $(69,31 \pm 7,05 \mathrm{~cm}) \mathrm{com}$ o esquerdo $(69,60 \pm 7,59 \mathrm{~cm}, \mathrm{p}=0,5258)$. $\mathrm{Na}$ comparação entre os grupos também não houve diferença significativa $(p>0,05)$ no Score Composto, assim sugerido o reteste como forma de avaliar o desempenho funcional e reavaliação do controle neuromuscular. Já no estudo de Artioli et al. (2011), os quais avaliaram indivíduos submetidos a tratamento fisioterapêutico pós-reconstrução do ligamento cruzado anterior, obtiveram como valores médios do Score Composto para o membro lesionado 73,66 $\pm 8,13$ $\mathrm{cm}$ e no membro inferior sadio de $74,43 \pm 8,31 \mathrm{~cm}$. Para o grupo controle ao comparar os lados direito e esquerdo, não houveram alterações significantes ( $\mathrm{p}$ $>0,05$ ). A média geral no MID foi de 71,12 $\pm 7,46 \mathrm{~cm}$ e no MIE de 71,52 $\pm 8,22$ $\mathrm{cm}$, sendo que comparando os grupos, não houve diferença significativa.

Já Manha, Silva e Fusaro (2018) avaliaram o controle sensório motor com o YBT em mulheres praticantes de corrida. Foi encontrada uma correlação positiva moderada de Pearson entre o tempo de prática e os Scores Compostos dos membros dominantes $r=0,4701$ e $p=0,0422$ e não dominantes $r=0,4743$ e $p=$ 0,0402 e uma correlação positiva fraca de Pearson entre o tempo de prática e a soma das medidas de alcance do membro dominante $r=0,3774$ e $p=0,1226$ e não dominante $\mathrm{r}=0,3838$ e $\mathrm{p}=0,1159$.

\section{CONCLUSÃO}

A incidência de lesões no meio esportivo é um tema preocupante, em boa parte devido ao aumento de jovens inseridos na prática. Além disso, um fator de aumento das lesões é devido ao mau planejamento e falta de avaliações associadas a predição de lesões. Os resultados aqui encontrados suportam a ideia de que testes funcionais são fundamentais para a identificação de futuras lesões em atletas, ainda mais considerando sua realização no período inicial da temporada. O YBT é um teste confiável, seguro e de baixo custo, portanto uma boa opção.

Pode-se concluir que o objetivo do presente estudo foi alcançado, identificando e comparando os desequilíbrios funcionais de MI nas avaliações pré e pós período de preparação. As diferenças encontradas foram poucas e não podem ser explicadas somente a partir dos valores aqui obtidos, sugerindose assim, futuras investigações sobre os tipos de treinamento utilizados para prevenção de lesões e equilíbrio funcional em atletas de voleibol de base..

\section{REFERÊNCIAS}

ANTÔNIO, V. de S.; SANTOS, M. A. G. N. dos. Prevalência de lesões em atletas de voleibol feminino e possíveis relações com treinamento inadequado e estresse. Revista Hórus, São Paulo, v. 7, n. 1, p. 57-69, 2012. 
ARTIOLI, D. P. et al. Teste de controle neuromuscular em indivíduos submetidos à reconstrução do ligamento cruzado anterior e em tratamento usioterapêutico avançado. Rev Bras Clin Med, São Paulo, v. 9, n. 4, p. 269-273, 2011.

BUTLER, R. J. et al. Dynamic Balance Performance and Noncontact Lower Extremity Injury in College Football Players: An Initial Study. Sports Health, v. 5, n. 5, p. 417422, 2013.

CANAVAN P. Reabilitação em medicina esportiva. 1. ed. São Paulo: Manole, 2001.

CORREA, D. R.; LINDEN JUNIOR, E. Avaliação do controle postural dinâmico em atletas amadoras de voleibol antes e após treinamento. Revista Simpósio de Fisioterapia - Uniplac, v. 3, p. 164-170, 2016.

COTTA, R. et al. Utilização dos testes de salto vertical e salto horizontal para prescrição de treinamento pliométrico. Revista Digital. v. 14, n. 131, p. 1-8, 2008. Disponível em: <http:/ / www.efdeportes.com/efd131/prescricao-de-treinamentopliometrico.htm>. Acesso em: 23 jun. 2018.

DALLINGA, J.M.; BENJAMINSE, A.; LEMMINK, K.A. Which screening tools can predict injury to the lower extremities in team sports?: a systematic review. Sports Med. v. 42, n. 9, p. 791-815, 2012.

FARINA, E. C. R. Risco de lesões na região do tornozelo em jogadores de voleibol: proposta de prevenção. EF y Deportes. Buenos Aires, v. 12, n. 117, 2008. Disponível em: <http:/ / www.efdeportes.com/efd117/riscos-de-lesoes-na\%20regiao-dotornozelo-em-19 jogadores-de-voleibol.htm>. Acesso em: 30 mai. 2018.

FILIPA, A. et al. Neuromuscular Training Improves Performance on the Star Excursion Balance Test in Young Female Athletes. Journal Of Orthopaedic \& Sports Physical Therapy, [s.1.], v. 40, n. 9, p.551-558, set. 2010. Journal of Orthopaedic \& Sports Physical Therapy (JOSPT). Disponível em: <http:/ /dx.doi.org/10.2519/ jospt.2010.3325>. Acesso em: 30 mai. 2018.

FRANCA, D; FERNANDES, V. S; CORTEZ, C. M. Acupuntura cinética como efeito potencializador dos elementos moduladores do movimento no tratamento de lesões desportivas. Revista Fisioterapia Brasil, v. 5, n. 2, p. 111- 118, 2004.

GOMES, A. C. Treinamento desportivo: Estruturação e periodização. 2. ed. Porto Alegre: Artmed, 2009.

GONELL, A. C.; ROMERO, J. A. P.; SOLER, L. M. RELATIONSHIP BETWEEN THE Y BALANCE TEST SCORES AND SOFT TISSUE INJURY INCIDENCE IN A SOCCER TEAM. The International Journal Of Sports Physical Therapy, v. 10, n. 7, p. 955-966, 2015.

GRIBBLE, P. A.; HERTEL, J.; PLISKY, P. Using the Star Excursion Balance Test to Assess Dynamic Postural-Control Deficits and Outcomes in Lower Extremity Injury: A Literature and Systematic Review. Journal Of Athletic Training, [s.1.], v. 47, n. 3, 
p. 339-357, maio 2012. Journal of Athletic Training/NATA. Disponível em: <http:/ / dx.doi.org/10.4085/1062-6050-47.3.08>. Acesso em: 30 mai. 2018.

HALE, S. A.; HERTEL, J.; OLMSTED-KRAMER, L. C.. The Effect of a 4-Week Comprehensive Rehabilitation Program on Postural Control and Lower Extremity Function in Individuals With Chronic Ankle Instability. Journal Of Orthopaedic \& Sports Physical Therapy, v. 37, n. 6, p. 303-311, 2007.

HERTEL, J.; BRAHAM, R. A.; HALE, S. A.; OLMSTED-KRAMER, L. C. Simplifying the Star Excursion Balance Test: Analyses of Subjects With and Without Chronic Ankle Instability. Journal Of Orthopaedic \& Sports Physical Therapy, v. 36, n. 3, p. 131-137, 2006.

HOOTMAN, J. M.; DICK, R.; AGEL, J. Epidemiology of Collegiate Injuries for 15 Sports:Summary and Recommendations for Injury Prevention Initiatives. Journal Of Athletic Training, v. 42, n. 2, p. 311-319, 2007.

JADHAV, K. G. et al. A Survey of Injuries Prevalence in Varsity Volleyball Players. Journal of Exercise Science and Physiotherapy, v. 6, n. 2, p. 102-105, 2010.

LOMBARDI, G.; VIEIRA, N. S.; DETANICO, D. Efeito de dois tipos de treinamento de potência no desempenho do salto vertical em atletas de voleibol. Brazilian Journal of Biomotricity, v. 5. n. 4. p. 230-238, 2011.

MAFFEY, L.; EMERY, C. PHYSIOTHERAPIST DELIVERED PREPARTICIPATION EXAMINATION: RATIONALE AND EVIDENCE. North American Journal Of Sports Physical Therapy: NAJSPT, v. 1, n. 4, p. 176-186, 2006.

MANCINI, M.; HORAK, F.B. The relevance of clinical balance assessment tools to differentiate balance deficits. Eur.J Phys Rehabil Med. v. 46, n. 2, p. 239-48, 2010.

MANHA, C. de C. S.; SILVA, G. M. G. da; FUSARO, C. Avaliação do controle sensório-motor com teste y-balance em mulheres praticantes de corrida de rua. 2018 . 41 f. Monografia (Graduação) - Curso de Fisioterapia, Universidade São Francisco, Bragança Paulista, 2018.

MARWAN, Y. et al. Sports Injuries among Professional Male Athletes in Kuwait: Prevalence and Associated Factors. Medical Principles And Practice, [s.1.], v. 21, n. 2, p. 171-177, 2012. S. Karger AG. Disponível em:

<http://dx.doi.org/10.1159/000332442>. Acesso em: 30 mai. 2018.

MATVEIEV, L. P. Fundamentos do treino desportivo. $2^{\mathrm{a}}$ ed. Lisboa: Livros Horizonte, p. 317, 1991.

PACHECO, I. CABRAL, S. Avaliação do uso de 3 diferentes tipos de tratamentofarmacológico na entorse de tornozelo em atletas. Revista Brasileira de Medicina do Esporte. v. 6, n. 5, p. 211, 2000. 
PLISKY, P. J. et al. Star Excursion Balance Test as a Predictor of Lower Extremity Injury in High School Basketball Players. Journal Of Orthopaedic \& Sports Physical Therapy, v. 36, n. 12, p. 911-919, 2006.

SILVA, K. M. A. da; ALMEIDA, T. G. de; REGIS, J. de F. Avaliação funcional do movimento em atletas de duas seleções de voleibol da região do vale do Jaguaribe/ CE. Boletim Informativo Unimotrisaúde em Sociogerontologia, Ceará, v. 8, n. 1, p. 3-27, 2017.

SMITH, C. A.; CHIMERA, N. J.; WARREN, M. Association of y balance test reach asymmetry and injury in division I athletes. Medicine and science in sports and exercise, v. 47, n. 1, p. 136-141, 2015.

TUBINO, M. J. G.; MOREIRA, S. B. Metodologia Científica do Treinamento Desportivo. 13. ed. Rio de Janeiro: Shape, 2003. 